\title{
Género, coautorías, e impacto: las publicaciones de investigadores peruanos en biología (1994 - 2017)
}

\author{
Lucía Málaga-Sabogal*, Francisco Sagasti* \\ *Universidad del Pacífico, Lima, Perú \\ Correo-e: lucisabogal@yahoo.es | ORCID iD: https://orcid.org/0000-0001-6346-5049 \\ Correo-e: f.sagastih@up.edu.pe | ORCID iD: https://orcid.org/0000-0003-2629-7351
}

Recibido: 01-09-19; 2a versión: 03-02-20; Aceptado: 04-02-20; Publicado: 02-03-21

Resumen: Este estudio explora el papel del género en las redes de coautoría, el impacto de los artículos escritos por dos o más autores, y la influencia de los artículos de alto impacto en las carreras académicas de los investigadores. Para este fin considera a los investigadores de biología peruanos con publicaciones en la colección principal de Web of Science y en el índice de citaciones de SciELO, así como las hojas de vida de los autores de publicaciones con mayor impacto. Se encontró un significativo desbalance en la cantidad de hombres y mujeres investigadores que publican, y que las mujeres tienden a publicar menos. No se detectaron preferencias de género en las redes de coautoría, pero sí que las mujeres ocupan en menor medida posiciones de alta intermediación, aquellas que son claves para direccionar el flujo de información en estas redes. Por último, el haber publicado una investigación de alto impacto se relaciona con el ascenso para los investigadores jóvenes, tanto hombres como mujeres.

Palabras clave: coautoría; impacto; género; biología; bibliometría

\section{Gender, coauthorship and impact: publications of Peruvian biology researchers (1994- 2017)}

Abstract: This study explores the role of gender in the networks of co-authorship publications, the impact of articles written by two or more authors, and the influence of high-impact articles on the academic careers. We analyze Peruvian biology researchers with publications in Web of Science Core Collection and SciELO Citation Index, as well as the curricula of the authors of greatest impact publications. A significant imbalance has been found in the number of male and female researchers as well as a significant disproportion of the number of documents published. The co-authorship network formation is not affected by gender, but women have high betweenness levels less frequently, which probably affects their effect on information flow. Finally having published a high impact research is related to promotion for junior researchers, both men and women.

Keywords: coauthorship; impact; gender; biology; bibliometrics

Cómo citar este artículo/Citation: Málaga-Sabogal, L.; Sagasti, F. (2021). Género, coautorías, e impacto: las publicaciones de investigadores peruanos en biología (1994 - 2017). Revista Española de Documentación Científica, 44 (1), e283. https://doi. org/10.3989/redc.2021.1.1707

Copyright: (C) 2021 CSIC. Este es un artículo de acceso abierto bajo los términos de la licencia de uso y distribución Creative Commons Reconocimiento 4.0 Internacional (CC BY 4.0). 


\section{INTRODUCCIÓN}

Numerosas investigaciones muestran que la coautoría influye positivamente en el impacto de un artículo científico medido por el número de veces que es citado (Adams, 2013; Torres-Salinas y otros, 2011). Los objetivos de este estudio son corroborar si hay alguna variación entre investigadores hombres y mujeres, y si el mayor impacto de las publicaciones con coautoría tiene relación con mejoras en sus carreras académicas.

Existe una correlación positiva entre la coautoría, especialmente internacional, y el número de citas que recibe un artículo (Adams, 2013). Participar en grupos de investigación con miembros de varios países ayuda a lograr una mejor recepción de los resultados publicados. La decisión sobre qué investigadores incorporar a un equipo internacional de investigación está generalmente, a cargo de investigadores principales que actúan como "gatekeepers" (Thege y otros, 2014), quienes consideran sus posibles aportes cognitivos y sociales, su capacidad de investigación y su potencial de desarrollo. El investigador joven interesado en formar parte de un equipo internacional evalúa las ventajas que le acarrea su participación, el prestigio de los futuros colaboradores, el número de lectores que estos pueden atraer, y su pertenencia a colegios invisibles que estudian temas de su interés (Melin, 2000). Además de lazos sociales tales como amistades de largo plazo o experiencias de interacción armoniosa, el género del investigador puede jugar un papel importante en la selección de colaboradores en equipos de investigación (Stvilia y otros, 2017; Carr y otros, 2009).

Hay varios estudios en los que una mayor proporción de hombres que mujeres publica en solitario (West y otros, 2013; Eigenberg y Whalley, 2015; Zettler y otros, 2017; Fell y König, 2016; Mcdowell y otros, 2006; Abramo y otros, 2013; Ozel y otros, 2014), aunque se registran excepciones (Corley y Sabharwal, 2010; Boschini y Sjögren, 2007). Sin embargo, esta dinámica probablemente esté en un proceso de transformación, ya que la proporción de textos en coautoría está aumentando a nivel mundial y en todas las disciplinas.

La preferencia de los investigadores para trabajar en equipo con personas de su propio sexo se considera frecuentemente como un supuesto subyacente a la participación femenina en un mundo académico predominantemente masculino (Zuckerman y otros, 1991). En algunas disciplinas y circunstancias se ha demostrado que esto es cierto (Teele y Thelen, 2017; Fahmy y Young, 2016; Boschini y Sjögren, 2007). Sin embargo, también hay casos, en los que el resultado no corrobora este postulado (Ozel y otros, 2014). Algunos autores se aventuran a suponer que estas diferencias pueden deberse a factores como rango académico y productividad, y que una vez se controlan estos factores las diferencias no son significativas (Aksnes y otros, 2019).

Hay diferencias de género en el desempeño académico de los investigadores, y éstas son evidentes en las publicaciones científicas. Las mujeres están en desventaja por su menor productividad medida a través del número de publicaciones (West y otros, 2013; Eigenberg y Whalley, 2015; Teele y Thelen, 2017; Corley y Sabharwal, 2010; Ozely y otros, 2014; Fahmy y Young, 2016; Ferber y Teiman, 1980; Maske y otros, 2003), o por el menor impacto de sus publicaciones (Ferber y Teiman, 1980; Maliniak y otros, 2013). ${ }^{1}$ Aunque hay quienes argumentan que estas desigualdades podrían explicarse, en mayor medida, por las auto-citas y el prestigio de las revistas en las que publican, más que por el género (Andersen y otros, 2019). Según Alcázar y otros (2018) las "desigualdades se expresan ... en el acceso y la movilidad en el espacio laboral, en la disparidad salarial, en la continua erosión de la presencia de mujeres en la carrera académica y en su estancamiento en puestos de menor nivel, así como en diferencias en la producción y visibilidad del conocimiento producido por hombres y mujeres".

Según las investigadoras, las dos razones fundamentales que explican estas diferencias son la concepción de un "académico ideal" ajustado a los modelos de comportamiento masculino, y la división sexual del trabajo doméstico y de cuidados. En el imaginario social los hombres tienden a ser dominantes, agresivos, racionales, independientes, emocionalmente despegados, y las mujeres sumisas, comprensivas, emocionales, dependientes y cariñosas. Las características de un "académico ideal" concuerdan con el estereotipo del carácter masculino, y afectan la percepción de desempeño de las mujeres en la academia. Además, la menor asertividad de las mujeres puede ocasionar compromisos que generan una gran carga laboral pero que llevan a poco reconocimiento, como por ejemplo ocurre en los casos de varias tareas de gestión (Mitchell y Hesli, 2013; Duran-Bellonch y Ion, 2014).

Más allá de los estereotipos del académico ideal, un mecanismo de discriminación adicional que interviene en el caso de las investigadores mujeres es la división sexual del trabajo doméstico y de cuidados (Mirick y Wladkowski, 2018; Angervall y Beach, 2017). Como explica Lorente Acosta (2017): 
El problema de la maternidad y el trabajo persiste en cuanto que su esencia no radica en si las mujeres deben contar con más o menos tiempo de permiso de maternidad, y si éste debe ser más o menos retribuido. El problema está en mantener la identidad de las mujeres sobre la maternidad nuclear, y en entender que todo lo que derive de ella es una consecuencia de su condición de mujer y una responsabilidad a la que no deben faltar. Todo lo demás gira alrededor de ese pivote, y si quieren trabajar fuera del hogar se les dice que es una decisión que ellas toman libremente y que, por tanto, deben de atenerse a esas consecuencias de afrontar una doble jornada. Los hombres, padres de esos mismos hijos (...) no son llamados por la cultura a ese encuentro con la responsabilidad paterna, es cierto que pueden acudir (...), y que muchos lo han hecho y lo hacen cada vez más, pero su identidad como hombres no está centrada en el cuidado y el afecto, sino en la protección y el mantenimiento material de la familia. (p. 185).

Si bien la menor productividad académica de las investigadoras mujeres es un fenómeno mundial, las diferencias culturales y académicas regionales y locales condicionan el grado y la manera en que se manifiesta. El contexto académico peruano es particularmente desfavorable para las mujeres: en el Perú sólo uno de cada tres investigadores es mujer, la menor proporción de investigadoras de América Latina (OCTS-OEI 2018)

\section{METODOLOGÍA Y FUENTES DE INFORMA- CIÓN}

Medicina y biología son las dos disciplinas con más publicaciones de investigadores afiliados a instituciones peruanas. Medicina ya cuenta con algunos estudios de coautorías, aunque no están centrados en las diferencias de género (Huamaní y Mayta-Tristán, 2010). En la presente investigación se escogió biología para examinar los patrones de desigualdad de género en una disciplina de las ciencias básicas, y se utilizaron indicadores de productividad, medida a través del número de publicaciones de cada autor; impacto, medido a través del número de citas que recibe un artículo; y el índice $\mathrm{H}$, que combina medidas de impacto y de productividad.

Para estudiar la información se empleó análisis de redes sociales. Cada autor es simbolizado por un vértice y la existencia de una relación entre los autores se señala con una arista. Otro concepto a tomar en cuenta es el camino - es a una secuencia de vértices dentro de una red tal que exista una arista entre cada vértice y el siguiente. La red suele dividirse en varias componentes conexas: subredes en las que existe un camino entre cualquiera de sus vértices.
A partir de las redes es posible obtener medidas de centralidad que describen la importancia de los vértices. El grado de un vértice en una red de coautorías corresponde al número de coautores que tiene el autor que representa el vértice. Con frecuencia es posible identificar un reducido número de investigadores que tienen un grado muy alto, que se denominan hubs. La intermediación refleja el control que ejerce un autor sobre el flujo de información en la red, y para analizarla se toma en cuenta sólo a los autores que forman parte de la componente gigante, es decir la componente conexa de mayor tamaño en la red. Esta medida no describe lo bien conectado que esta un autor, sino la frecuencia con que intermedia entre otros autores, por lo que algunos autores podrían tener un grado relativamente bajo, pero una intermediación relativamente alta. A estos autores se les denomina brokers o gatekeepers por su capacidad de controlar el acceso a la red y porque su remoción podría afectar la fluidez de la comunicación en ella.

Por último, la asortatividad mide la tendencia de los vértices a vincularse con aquellos que tienen características análogas (Newman, 2010; Kumar, 2015). Cuando los autores representados por los vértices tienden a relacionarse sólo con otros de características similares la red es totalmente asortativa (valor 1 ), cuando tienden a relacionarse sólo con aquellos de características diferentes la red es totalmente disortativa (valor -1), y es parcialmente asortativa cuando esta medida se ubica en un rango intermedio (Barabási, 2014).

En este estudio el análisis de los datos bibliográficos abarcó la identificación de los autores, su género, productividad, impacto y centralidad en la red de coautorías, además de evaluar la tendencia de autores del mismo sexo a trabajar entre ellos (medidas de asortatividad). La información y los datos analizados provienen de una búsqueda en revistas categorizadas en biología en Web of Science Core Collection y en SciELO Citation Index, realizada el 30 de noviembre 2017. Se buscó Perú en el campo «CU». Se encontraron 894 artículos, publicados entre 1994 y 2017 por 1755 autores. Para el análisis se usó el software estadístico R. Además de las funciones que vienen con el programa se usaron varios paquetes adicionales: readr, readxl, stringr, para la lectura y formateo de datos, stringdist para desambiguación de autores, gender y genderizeR para reconocimiento de género, igraph, para el análisis de redes y la visualización. La desambiguación de autores se realizó utilizando la distancia q (q-gram) y se complementó con revisión manual. El reconocimiento de género se dio a partir del primer nombre. En los casos en los que hubo dudas se hizo una búsqueda de información sobre los autores en internet. 
Si aún así no se contaba con suficiente información se asignó género desconocido.

La relación de las publicaciones con la carrera académica se estudió en base a las hojas de vida de los investigadores de los equipos que publicaron los diez artículos con mayor impacto en los últimos diez años. La información se recopiló del Directorio Nacional de Investigadores (DINA) del Consejo Nacional de Ciencia y Tecnología (CONCYTEC). En los casos en que esto no fue suficiente, se obtuvo información adicional de LinkedIn e instituciones donde trabajan o trabajaron los autores, para información laboral. Se tomó en cuenta un total de 28 investigadores asociados a instituciones peruanas $^{2}$. Además, fue posible realizar un breve cuestionario a once investigadoras de las 23 investigadoras con mayor índice $\mathrm{H}$. Se les preguntó el grado máximo alcanzado, la fecha de graduación, edad, año en el que tuvieron al primer hijo y el número total de hijos.

Las pruebas estadísticas fueron: prueba de independencia, prueba de Kolmogorov - Smirnov y coeficientes de correlación de Kendall y de Spearman.

\section{RESULTADOS}

\subsection{Presencia de mujeres}

El $37 \%$ de los 1.733 investigadores, cuyo género se pudo identificar, son mujeres. Esta proporción es significativamente superior al $23 \%$ de investigadores mujeres que publicaron artículos citados en las áreas de ecología, evolución, biología molecular y biología celular en el ámbito mundial (West y otros, 2013).

Al igual que en otros países, las mujeres publican menos que los hombres. Entre los 33 autores con más de seis artículos, sólo $18 \%$ son mujeres, en comparación con el $37 \%$ de mujeres entre el total de autores. Por otra parte, el $68 \%$ de los investigadores hombres han publicado un solo artículo, pero este porcentaje es mayor (75\%) en el caso de investigadores del género femenino. Además, un análisis de los trece autores con mayor productividad en el componente gigante de la red de coautorías muestra que sólo tres son mujeres (figura 1 ).

Podría suponerse que la maternidad explica las diferencias en los patrones de autoría entre in-

Figura 1. Autores con mayor productividad en la componente gigante.

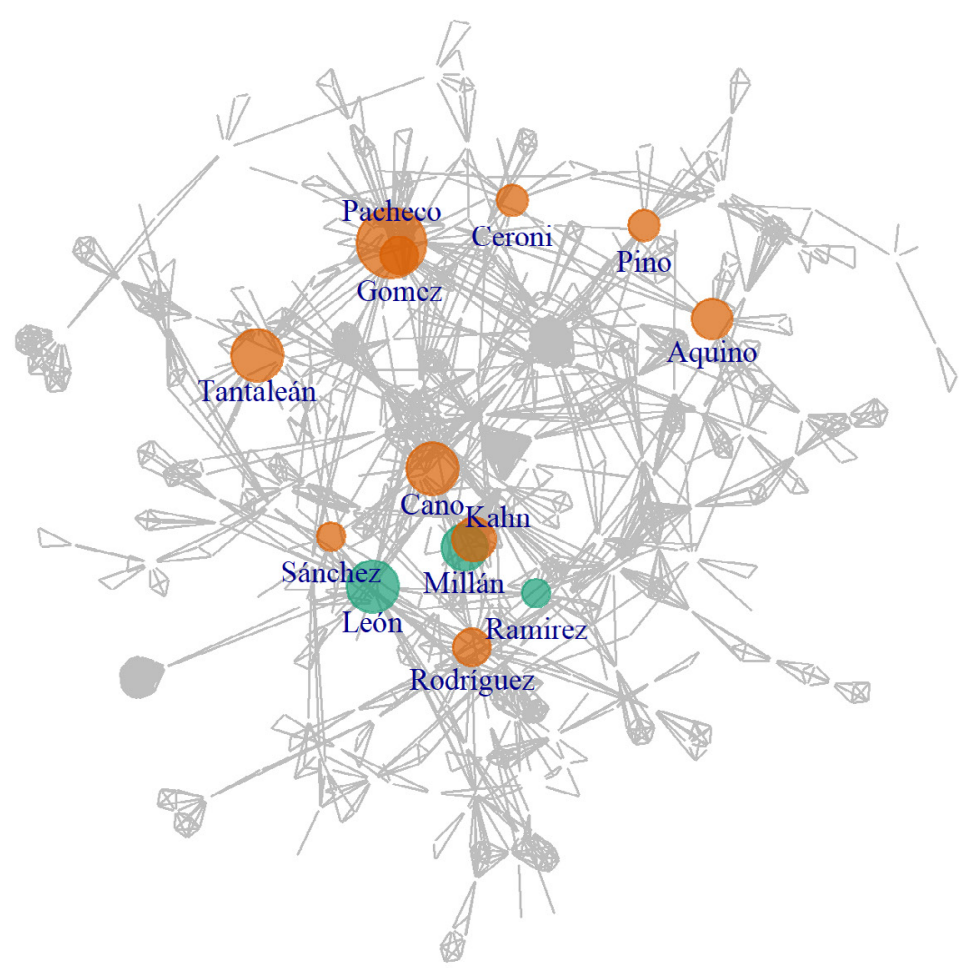

Nota: Se muestra a los autores con diez o más publicaciones que forman parte de la componente gigante. En naranja a los hombres y en verde a las mujeres. El tamaño de los vértices es proporcional al número de publicaciones del autor. En segundo plano se visualizan todas las aristas del componente principal.

Ver características de los autores en el anexo 2. 
vestigadores hombres y mujeres, pero estudios del grupo Sofía sobre investigadoras peruanas en ciencias sociales muestran que, incluso cuando no tienen infantes a su cuidado, las mujeres le dedican más tiempo que los hombres a las tareas del hogar (Alcázar y otros, 2018). Los resultados sugerirían que las mujeres abandonan la carrera de investigación con mayor frecuencia que los hombres, o que las mujeres que continúan su carrera académica estarían dedicándose más a actividades que no culminan en artículos académicos. En el caso de las investigadoras de la Facultad de Biología de la Universidad Nacional Mayor de San Marcos, que es la institución con mayor presencia entre las estudiadas, tanto la decana como las dos vicedecanas son mujeres y, como tales, tienen una sustantiva carga de tareas de gestión. Sin embargo, conocer si las mujeres, en general, se dedican en mayor medida a actividades de gestión amerita un estudio aparte.

\subsection{Coautoría}

El análisis de los datos muestra que, contrariamente a la hipótesis inicial de trabajo, la colaboración entre los autores peruanos en biología no depende del género: ni los varones tienden a escribir con varones ni las mujeres con mujeres. Las medidas de asortatividad son cercanas a cero en ambos casos: 0.03 para mujeres y 0.02 para hombres. Tampoco hay evidencia de desigualdad de género en el grado o número de coautores que tienen los investigadores.

\subsection{Impacto}

No se han encontrado diferencias significativas en cuanto al porcentaje de artículos publicados por equipos de investigación sólo masculinos, sólo femeninos o mixtos que se encuentran en el grupo del $10 \%$ de los artículos más citados (más detalles en Tabla 1 en anexo).

Además del impacto medido a través de citaciones, las mujeres pueden ejercer poder sobre el flujo de información dependiendo de su posición en la red de coautorías. La capacidad de posicionarse como broker o intermediario en una red de publicaciones científicas está correlacionada con la productividad de los autores, ${ }^{3}$ y la menor productividad de las mujeres investigadoras podría afectar su capacidad de actuar como brokers. Para el análisis de intermediación en la red de investigadores peruanos en biología se consideró sólo la componente gigante, en la cual $39 \%$ de los autores son mujeres; pero si se toma en cuenta sólo el $10 \%$ de autores con más intermediación, este porcentaje se reduce a $27 \%$.
Como se indicó anteriormente, el índice $\mathrm{H}$ combina en una sola medida la productividad y el impacto de las publicaciones de los investigadores. Es de esperar que la menor productividad de las mujeres influya en el valor de este índice: si bien el $36 \%$ de todos los autores estudiados son mujeres, sólo se tiene un $18 \%$ de mujeres entre los 22 autores con índice $\mathrm{H}$ mayor a 2.

Como puede verse el alto impacto es una medida muy relativa. En líneas generales los valores de citación y el índice $\mathrm{H}$ hallados son muy bajos. Si bien no contamos con exactamente los mismos datos para otros países de la región, sí tenemos los datos del promedio de citas por artículo, y el único país de la región ${ }^{4}$ que tiene un promedio de citas muy superior al peruano es Brasil con 2.41 citas por artículo. Los otros países de la región tienen promedios cercanos al 1.43 peruano. México tiene 1.18, Argentina 1.19, Venezuela 1.33, Costa Rica 1.46, Colombia 1.68 .

\subsection{Carreras académicas}

Para explorar la relación entre las carreras académicas y la participación en equipos de investigación de artículos de alto impacto se examinaron las hojas de vida de los 24 autores afiliados a instituciones peruanas que publicaron diez artículos altamente citados durante los últimos diez años, y cuyas hojas de vida están disponibles en línea. ${ }^{5}$ Para determinar si las hojas de vida de estos investigadores con publicaciones de alto impacto fueron significativamente diferentes a las de investigadores que no tuvieron artículos altamente citados, se escogió además una muestra al azar de 28 investigadores no citados que publicaron en el mismo periodo que los autores de artículos de alto impacto, 22 de los cuales tenían sus hojas de vida disponibles en línea. Empleando información provista por los investigadores se identificó si el autor había recibido promoción laboral, definida como un nuevo cargo de profesor, cargo de investigador o una mejora laboral, en el transcurso de los cuatro años siguientes a la publicación de un artículo. En ambos grupos de investigadores se detectó avances en la carrera académica en cerca del $50 \%$ de los casos, sin que existan diferencias significativas entre quienes escribieron artículos de alto impacto y quienes no lo hicieron. ${ }^{6}$

Una segunda aproximación a la relación entre la publicación de un artículo de alto impacto y la carrera académica del investigador se centró en las características de la publicación que intentan determinar si el artículo es particularmente importante para la trayectoria del investigador: se consideraba que esto era cierto (1) si era su primer artículo de 
alto impacto, y (2) si era resultado de una investigación realizada como parte de sus estudios de posgrado. Se estudió a los mismos 24 investigadores autores de artículos altamente citados y se los separó en dos grupos: aquellos para los que el artículo fue particularmente importante y aquellos para los que fue uno de los muchos artículos altamente citados. Se encontró evidencia de que existe relación entre la publicación de un artículo de alto impacto, que cumple con alguna de esas dos condiciones, y el avance en la carrera académica. El $80 \%$ de los investigadores cuyos artículos de alto impacto eran importantes tuvo acceso a mejores oportunidades laborares, mientras que entre los investigadores que publicaron artículos de alto impacto, pero no cumplieron con ninguna de estas condiciones, sólo el $43 \%$ tuvieron una mejora laboral.

\subsection{Carrera académica y género}

Se comparó el desempeño de las siete investigadoras mujeres y de los 17 investigadores hombres que participaron en los equipos de investigación de los diez artículos más citados de la muestra y cuyas hojas de vida están disponibles en línea. No se encontró diferencias significativas en cuanto a las oportunidades laborales de unos y otros.

Se procedió a explorar la relación entre las carreras académicas y la participación en equipos de investigación de artículos de alto impacto de las siete mujeres que participaron en los equipos de investigación que publicaron diez artículos altamente citados durante los últimos diez años, y cuyas hojas de vida están disponibles en línea. Se comparó su desempeño con el de catorce investigadoras seleccionadas al azar que escribieron en el mismo periodo, pero cuyos artículos no fueron citados. Al igual que se hizo en la etapa anterior, empleando información provista por los investigadores se identificó si el autor había recibido promoción laboral. En ambos grupos de investigadores se detectó avances en la carrera académica en más del $40 \%$ de los casos, sin que existan diferencias significativas entre quienes escribieron artículos de alto impacto y quienes no lo hicieron.

Adicionalmente, de las 23 mujeres investigadoras con un índice $\mathrm{H}$ de dos o más afiliadas a instituciones peruanas, ${ }^{7}$ once respondieron un cuestionario sobre su maternidad y grados académicos. Estas investigadoras tienen actualmente entre 48 y 63 años, seis no tuvieron hijos, cuatro tuvieron un hijo, una tuvo dos hijos, y todas ellas tuvieron sus hijos entre los 28 y 39 años, En contraste, el $57 \%$ de mujeres con maestría o doctorado de ese rango de edad tiene dos o más hijos (Censo Nacional 2017 de Población, Vivienda y Comunidades In- dígenas), y en el área urbana la mediana de edad para tener el primer hijo es 23.1 años (Encuesta Demográfica y de Salud Familiar 2017) .

No olvidemos que la aplicación de pruebas estadísticas tiene sus limitaciones y el que no se haya hallado evidencia de una relación no significa que esa relación no exista (Amrhein y otros, 2019). Por otro lado, en este estudio se ha considerado estadísticamente significativas pruebas con valores de $\mathrm{p}$ hasta 0.1 . En todos los casos los resultados se detallan en la Tabla I para mejor juicio del lector.

\section{DISCUSIÓN}

La desigualdad de género en el mundo académico peruano es importante y en ninguna de las áreas de investigación el número de mujeres es igual al número de hombres. No obstante, esta desproporción es muy variable: en medicina es pequeña, en las ciencias físicas y matemáticas es muy pronunciada, y la presencia femenina en disciplinas vinculadas a la ingeniería ocupa un lugar intermedio entre ambos extremos. Sería recomendable realizar estudios similares al presente en otras disciplinas para corroborar los resultados obtenidos en biología, particularmente con referencia al papel que juega la publicación en coautoría, especialmente internacional, en la visibilidad y la carrera de las mujeres investigadoras.

Se identificaron varios casos de investigadoras exitosas en el mundo académico peruano que tienen tanto productividad como números de citaciones por encima de la media. No existe suficiente información para conocer con precisión las razones por las cuales estas investigadoras se desempeñaron con éxito en un ambiente que, aparentemente, favorece a los hombres. Sin embargo, existen indicios de que estas investigadoras postergaron la maternidad para mejorar su desempeño académico. En base al cuestionario realizado sabemos que las mujeres que alcanzaron cierto éxito académico han tenido menos hijos y los han tenido más tarde que otras. La relación entre decisiones sobre maternidad y desempeño académico de las investigadoras merece mayor estudio y análisis, ya que el cuestionario que se aplicó fue breve y sólo abarcó a la mitad de las autoras más importantes.

Por otra parte, existen diversas experiencias de evaluación de desempeño de investigadores que gradualmente se están implementando en Perú. Los resultados de este estudio se enmarcan en el contexto de incipientes prácticas de evaluación aplicadas a los miembros de la comunidad científica, cuyo comportamiento aún no ha sido afectado por la implementación de un sistema de calificación de los investigadores. 
Tabla I. Características de los investigadores peruanos en biología

\begin{tabular}{|c|c|c|}
\hline Característica & Evidencia & Prueba (si aplica) \\
\hline \multicolumn{3}{|c|}{ Pregunta de investigación: ¿Cuáles son las características de las investigadoras mujeres? } \\
\hline $\begin{array}{l}\text { Menos investigadoras } \\
\text { mujeres }\end{array}$ & $\begin{array}{l}638 \text { investigadoras mujeres ( } 37 \% \text { del total de autores cuyo } \\
\text { sexo se identificó) }\end{array}$ & \\
\hline \multirow{3}{*}{$\begin{array}{l}\text { Las mujeres tienen } \\
\text { menor productividad } \\
\text { (número de artículos) }\end{array}$} & $\begin{array}{l}\text { Diferentes distribuciones de productividad para hombres y } \\
\text { mujeres }\end{array}$ & $\begin{array}{l}\text { Prueba de } \\
\text { Kolmogorov-Smirnov } \\
\text { p-value }=0.05472\end{array}$ \\
\hline & $\begin{array}{l}\text { Entre los } 33 \text { autores con más de seis documentos (el } 2 \% \text { de } \\
\text { autores más productivos), sólo } 18 \% \text { son mujeres versus } 36 \% \\
\text { de mujeres entre el total de autores. }\end{array}$ & $\begin{array}{l}\text { Prueba Chi cuadrado } \\
\text { X-squared = } 4.0818 \\
\text { df }=1, p \text {-value }= \\
0.04335\end{array}$ \\
\hline & $\begin{array}{l}68 \% \text { de investigadores hombres han escrito un solo artículo, } \\
75 \% \text { de mujeres han escrito un solo artículo. }\end{array}$ & \\
\hline \multicolumn{3}{|c|}{ ¿Cuáles son las características de las mujeres investigadoras? } \\
\hline $\begin{array}{l}\text { No hay preferencia de los } \\
\text { hombres por publicar con } \\
\text { hombres ni de mujeres } \\
\text { por publicar con mujeres }\end{array}$ & Medidas de asortatividad cercanas a cero en ambos casos. & $\begin{array}{l}\text { Asortatividad para } \\
\text { las mujeres } \\
0.029 \\
\text { Asortatividad para } \\
\text { los varones } \\
0.022\end{array}$ \\
\hline \multicolumn{3}{|c|}{$\begin{array}{l}\text { Pregunta de investigación: ¿EI impacto alcanzado por las mujeres es diferente al alcanzado por los } \\
\text { hombres? }\end{array}$} \\
\hline $\begin{array}{l}\text { No hay evidencia } \\
\text { de diferencias en el } \\
\text { impacto asociadas a la } \\
\text { composición de género } \\
\text { de los equipos }\end{array}$ & $\begin{array}{l}\text { Los equipos de investigación en su mayoría son mixtos o } \\
\text { exclusivamente masculinos. No hay evidencia que la presencia } \\
\text { de mujeres en el equipo tenga relación con el impacto } \\
\text { alcanzado. } 14 \% \text { de los } 223 \text { artículos escritos por equipos } \\
\text { masculinos y } 12 \% \text { de los } 502 \text { artículos escritos por equipos } \\
\text { mixtos está entre los } 10 \% \text { más citados }\end{array}$ & $\begin{array}{l}\text { Prueba Chi } \\
\text { Cuadrado } \\
\text { X-squared }= \\
0.47276, d f=1 \\
\text { p-value }=0.4917\end{array}$ \\
\hline \multirow{2}{*}{$\begin{array}{l}\text { Las mujeres se ven en } \\
\text { desventaja en el capital } \\
\text { social medido a través de } \\
\text { la intermediación }\end{array}$} & $\begin{array}{l}\text { El } 27 \% \text { de los } 82 \text { autores con más intermediación (decil } \\
\text { superior) son mujeres, en comparación con el } 39 \% \text { de mujeres } \\
\text { en la totalidad de la componente gigante. }\end{array}$ & $\begin{array}{l}\text { Prueba Chi cuadrado } \\
\text { X-squared }= \\
4.0555, \mathrm{df}=1 \\
\mathrm{p} \text {-value }=0.04403\end{array}$ \\
\hline & $\begin{array}{l}\text { Existe una correlación entre la intermediación y la } \\
\text { productividad de los autores, por lo que la menor productividad } \\
\text { de las mujeres investigadoras podría afectar su nivel de } \\
\text { intermediación }\end{array}$ & $\begin{array}{l}\text { Kendall } \\
\text { Tau }=0.5039418 \\
\text { Spearman } \\
\text { Rho }=0.6019769\end{array}$ \\
\hline $\begin{array}{l}\text { Menor presencia de } \\
\text { mujeres entre autores } \\
\text { con mayor índice H }\end{array}$ & $\begin{array}{l}\text { Entre los } 22 \text { autores con índice } \mathrm{H} \text { mayor a dos (percentil } \\
\text { superior), sólo hay } 18 \% \text { de mujeres versus } 36 \% \text { de mujeres en } \\
\text { el total de la población. }\end{array}$ & $\begin{array}{l}\text { Prueba exacta de } \\
\text { Fisher } \\
\text { p-value }=0.07691\end{array}$ \\
\hline \multicolumn{3}{|c|}{$\begin{array}{l}\text { Pregunta de investigación: ¿Las carreras académicas de los investigadores se ven afectadas por la } \\
\text { publicación de artículos de alto impacto? }\end{array}$} \\
\hline $\begin{array}{l}\text { No hay evidencia de } \\
\text { dependencia entre las } \\
\text { variables ascenso y } \\
\text { artículo de alto impacto }\end{array}$ & $\begin{array}{l}\text { Comparando las carreras académicas de un conjunto de autores } \\
\text { que escribió artículos de alto impacto, y de autores que escribieron } \\
\text { artículos que no fueron citados, no se aprecia evidencia de que el } \\
\text { impacto del artículo tenga relación con un posible ascenso. } 58 \% \\
\text { de los autores que escribieron artículos de alto impacto tuvieron } \\
\text { un ascenso, mientras que el } 48 \% \text { de una muestra de autores que } \\
\text { escribieron artículos que no fueron citados accedieron a un ascenso. }\end{array}$ & $\begin{array}{l}\text { Prueba Chi Cuadrado } \\
\text { X-squared }= \\
0.18426, \mathrm{df}=1 \\
\text { p-value }=0.6677\end{array}$ \\
\hline $\begin{array}{l}\text { Para los investigadores } \\
\text { jóvenes, la publicación } \\
\text { de un artículo de alto } \\
\text { impacto sí puede tener } \\
\text { relación con un ascenso }\end{array}$ & $\begin{array}{l}\text { Se separó a los autores de artículos de alto impacto en dos } \\
\text { grupos: (1) investigadores que ya tenían varios artículos de } \\
\text { alto impacto adicionales al analizado, ( } 2 \text { ) investigadores para } \\
\text { los cuales el artículo analizado era el primer artículo de alto } \\
\text { impacto; o era uno de los primeros más citados y al mismo } \\
\text { tiempo era resultado de una investigación de posgrado. Se } \\
\text { halló que sí había evidencia entre un ascenso y la pertenencia a } \\
\text { alguno de estos dos grupos. En el grupo } 1 \text { el } 80 \% \text { accedió a un } \\
\text { ascenso, en el grupo } 2 \text { el } 43 \% \text { accedio a un ascenso. }\end{array}$ & $\begin{array}{l}\text { Prueba exacta de } \\
\text { Fisher } \\
\text { p-value }=0.1\end{array}$ \\
\hline \multicolumn{3}{|c|}{$\begin{array}{l}\text { Pregunta de investigación: ¿Las carreras académicas de las mujeres se ven afectadas por la publicación } \\
\text { de artículos de alto impacto? }\end{array}$} \\
\hline $\begin{array}{l}\text { No hay evidencia de } \\
\text { dependencia entre la } \\
\text { variable género y la } \\
\text { variable carrera académica }\end{array}$ & $\begin{array}{l}\text { Comparando las carreras académicas de hombres que } \\
\text { escribieron artículos de alto impacto, y de mujeres que lo } \\
\text { hicieron, no se aprecia evidencia de que el género del autor } \\
\text { tenga relación con un posible ascenso. }\end{array}$ & $\begin{array}{l}\text { X-squared }= \\
0.6805, \mathrm{df}=1 \\
\text { p-value }=0.4094\end{array}$ \\
\hline
\end{tabular}


Entre diciembre del 2015 y noviembre del 2018 existió el Registro de Investigadores en Ciencia y Tecnología del Sistema Nacional de Ciencia, Tecnología e Innovación Tecnológica (REGINA). Era necesario estar registrado en REGINA para ser considerados docente-investigador en las universidades nacionales. Sólo los docentes investigadores tienen derecho a reducir sus horas de dictado para dedicarlas a la investigación, y a recibir una bonificación por ello. Un requisito para estar registrado en REGINA era haber escrito un artículo de investigación en una revista indizada en Scopus o Web of Science, o dos artículos en SciELO durante los últimos siete años. Además, era necesario alcanzar un puntaje mínimo y uno de los indicadores considerados para determinar este puntaje era el índice $\mathrm{H}$. En octubre del 2018, se modificó este sistema con la creación del Registro Nacional Científico, Tecnológico y de Innovación Tecnológica (RENACYT), que considera la publicación de artículos como criterio, pero que ya no toma en cuenta las citas. Esto sugiere la necesidad de estudiar la manera en que los patrones de coautoría y la carrera académica podrían cambiar tras la implementación del nuevo sistema de evaluación.

\section{CONCLUSIONES Y RECOMENDACIONES}

La coautoría es una práctica establecida entre los investigadores peruanos en biología que publican en revistas indexadas. Si bien la participación de coautores internacionales está asociada con un mayor impacto de los artículos medido en término de citaciones, la coautoría de artículos se da mayormente entre investigadores nacionales. Por otra parte, no se aprecia una preferencia por publicar con autores del mismo sexo, y los equipos de investigadores que publican son en su mayoría mixtos. La publicación de un artículo de alto impacto no tiene influencia en la carrera de los investigadores establecidos, pero podría tenerla en la carrera de los investigadores jóvenes autores de un artículo de alto impacto al inicio de su carrera.

Cuando mencionamos el alto impacto es una medida muy relativa. En líneas generales los valores de citación y el índice $\mathrm{H}$ hallados son muy bajos, aunque esto no difiere de los países vecinos, a excepción de Brasil. Los resultados alcanzados son una muestra más de que la región está en la periferia de la ciencia.

Las mujeres investigadoras están en desventaja en relación con sus pares del sexo masculino. Publican menos artículos y un mayor número de mujeres que de hombres son autoras de un solo artículo. Las mujeres tienen menor presencia entre los autores con alto nivel de intermediación que actúan como brokers, y también entre aquellos que tienen los índices $\mathrm{H}$ más altos. No se encontró evidencia de diferencias de género al examinar las carreras académicas de hombres y mujeres, pero el número de casos es muy pequeño para extraer conclusiones definitivas.

Los hallazgos de este estudio sugieren que, además de iniciativas generales para mejorar la participación femenina en la comunidad de investigadores en biología en el Perú, tales como incentivos y capacitación para participar en equipos de investigación, programas específicos de mentoría, estímulos para continuar publicando más allá de un primer artículo, y mayor flexibilidad laboral para mujeres en gestación, sería conveniente promover la publicación de artículos científicos con coautores internacionales.

La presencia de un coautor internacional en un artículo escrito por una investigadora mujer permitiría que su trabajo tenga mayor visibilidad e impacto, especialmente si se este artículo se publica al inicio de su carrera académica. Para ese fin sería apropiado promover la participación de investigadores mujeres en las redes internacionales de investigación, organizar pasantías postdoctorales en centros de investigación de otros países, promover proyectos de investigación con cooperación internacional en los que participen investigadores mujeres, y estimular financieramente la publicación de artículos escritos con académicos de otros países.

Ya que la menor productividad de las mujeres perjudica su posición en las redes y con frecuencia en el índice $\mathrm{H}$, varios recomiendan no tomar en cuenta este indicador para el futuro. Sin embargo, si existe la práctica de evaluación debemos utilizar algunas medidas y es probable que si optamos por cualquiera de ellas las mujeres se vean perjudicadas ya que hay razones de orden social, e incluso biológico que son factores de peso en la distribución del tiempo femenino que las ponen en desventaja. Por eso, creemos que el uso de indicadores de productividad en la evaluación debe realizarse con mucho cuidado.

Esta preocupación está presente en la academia hace ya un buen tiempo, como, por ejemplo, en la Declaración de San Francisco sobre la Evaluación de la Investigación (DORA) (2012). En este documento se recomienda que las agencias de financiación sean explícitas "sobre los criterios utilizados para evaluar la productividad científica de los solicitantes de fondos de investigación", ya que el contenido científico de un artículo es mucho más importante que las métricas de publicación o la identidad de la 
revista en la que fue publicado. Nosotros agregaríamos que en cualquier evaluación debe considerarse también la edad académica de los investigadores, definida como el tiempo que se ha dedicado a la investigación. Por eso, en el caso de las mujeres, el nacimiento de un hijo debería ser considerado como un receso, de como mínimo de un año y, en los casos que lo amerite, incluso más.

Si se considera este tipo de limitaciones el índice $\mathrm{H}$ podría no ser la medida más adecuada, pero debería considerarse en cada uno de los entornos si es el más idóneo. En contextos nacionales, y sin otros indicadores que lo complementen, no sería la mejor opción.

\section{NOTAS}

1. El impacto académico se mide a través del número de citas que recibe un artículo.

2. La mayoría de los investigadores no comparte información detallada sobre su trayectoria académica para periodos más largos.

3. En la prueba de correlación de Kendall el valor Tau es 0.5 , en la de Spearman, el Rho es 0.6.

4. Consideramos a los países con más de 600 artículos de biología publicados hasta el 2017.

5. La exigencia de hacer públicas las hojas de vida en el Directorio Nacional de Investigadores (DINA) del CONCYTEC es reciente y los investigadores rara vez cumplen con completar su trayectoria para un periodo mayor a diez años.

\section{REFERENCIAS}

Abramo, G.; D'Angelo, C. A.; Murgia, G. (2013). Gender differences in research collaboration. Journal of Informetrics, 7 (4), 811-822. https://doi.org/10.1016/j. joi.2013.07.002

Adams, J. (2013). Collaborations: The fourth age of research. Nature, 497 (7451), 557-560. https://doi.org/10.1038/497557a

Aksnes, Dag W., Fredrik Niclas Piro, y Kristoffer Rørstad. 2019. "Gender gaps in international research collaboration: A bibliometric approach". Scientometrics 120 (2): 747-74. https://doi.org/10.1007/s11192-019-03155-3.

Alcázar, L.; Balarín, M. (2018). ¿Cómo abordar el estudio de las inequidades de género en el mundo académico? En L. Alcázar \& M. Balarín (Eds.), Desigualdad en la academia: Mujeres en las ciencias sociales peruanas, pp. 25-36. Lima: Grupo Sofía y GRADE. https://www. grade.org.pe/publicaciones/desigualdad-en-la-academia-mujeres-en-las-ciencias-sociales-peruanas/

Alcázar, L.; Balarín, M.; Román, A. (2018). Oportunidades y barreras en la trayectoria de las científicas sociales peruanas. En L. Alcázar \& M. Balarín (Eds.), Desigualdad en la academia: Mujeres en las ciencias sociales peruanas, pp. 51-101. Lima: Grupo Sofía y GRADE. https://www. grade.org.pe/publicaciones/desigualdad-en-la-academia-mujeres-en-las-ciencias-sociales-peruanas/

Amrhein, V.; Greenland, S.; McShane, B. (2019). Scientists rise up against statistical significance. Nature, 567 (7748), 305-307. https://doi.org/10.1038/d41586-01900857-9
Por último, este estudio tiene limitaciones metodológicas, por un lado, la cantidad de investigadoras mujeres que tuvieron un impacto elevado dentro del universo estudiado es muy pequeña. Sólo siete mujeres afiliadas a instituciones peruanas participaron en la redacción de los diez artículos académicos más citados. Sólo 23 tienen un índice $\mathrm{H}$ de 2 o más, y de estas 23 sólo pudo hacerse cuestionarios sobre su maternidad a 11 . La poca presencia femenina entre los autores con mejor desempeño es una de las consecuencias de la desigualdad existente, pero también significa que los resultados aquí presentados deben tomarse con cautela ya que se trata de poblaciones pequeñas.

6. El que un artículo de alto impacto publicado por investigadores biólogos peruanos no tenga influencia en su carrera académica podría deberse a que, además de artículos académicos en biología, el investigador publica textos categorizados en otra disciplina (por ejemplo medicina) que han recibido un mayor número de citaciones; el autor ha escrito uno o varios artículos altamente citados con anterioridad al periodo estudiado; o a que el autor ha escrito textos altamente citados que no son cubiertos por las bases de datos de las que proviene la información analizada.

7. Es decir, investigadoras que tienen por lo menos dos artículos citados por lo menos dos veces cada uno.

Andersen, J. P.; Schneider, J. W.; Jagsi, R.; Nielsen, M. W. (2019). Gender variations in citation distributions in medicine are very small and due to self-citation and journal prestige. eLife, 8, e45374. https://doi. org/10.7554/eLife.45374

Angervall, P.; Beach, D. (2017). Dividing academic work: gender and academic career at Swedish universities. Gender and Education, 1-16. https://doi.org/10.1080 /09540253.2017.1401047

Barabási, A.-L. (2014). Degree Correlation. In Network Science. http://barabasi.com/f/620.pdf

Boschini, A.; Sjögren, A. (2007). Is team formation gender neutral? Evidence from coauthorship patterns. Journal of Labor Economics, 25 (2), 325-365. https:// doi.org/10.1086/510764

Carr, P. L.; Pololi, L.; Knight, S.; Conrad, P. (2009). Collaboration in academic medicine: Reflections on gender and advancement. Academic Medicine: Journal of the Association of American Medical Colleges, 84 (10), 14471453. https://doi.org/10.1097/ACM.0b013e3181b6ac27

Corley, E. A.; Sabharwal, M. (2010). Scholarly collaboration and productivity patterns in public administration: analysing recent trends. Public Administration, 88 (3), 627-648. https://doi.org/10.1111/j.1467-9299.2010.01830.x

Csardi, G.; Nepusz, T. (2006). The igraph software package for complex network research. InterJournal, Complex Systems, 1695.

Declaración de San Francisco sobre la Evaluación de la Investigación (2012). https://sfdora.org/ 
Duran-Bellonch, Mar y Georgeta Ion, 2014. "Investigadoras con éxito en la universidad... ¿Cómo lo han logrado?" Educación XX1 17 (1): 39-58. https://doi. org/10.5944/educxx1.17.1.10704

Eigenberg, H. M.; Whalley, E. (2015). Gender and publication patterns: female authorship is increasing, but is there gender parity? Women \& Criminal Justice, 25 (1-2), 130-144. https://doi.org/10.1080/08974454.2 015.1023882

Fahmy, C.; Young, J. T. N. (2016). Gender inequality and knowledge production in criminology and criminal justice. Journal of Criminal Justice Education, 28 (2), 285305. https://doi.org/10.1080/10511253.2016.1233346

Fell, C. B.; König, C. J. (2016). Is there a gender difference in scientific collaboration? A scientometric examination of co-authorships among industrial-organizational psychologists. Scientometrics, 108 (1), 113-141. https://doi.org/10.1007/s11192-016-1967-5

Ferber, M. A.; Teiman, M. (1980). Are women economists at a disadvantage in publishing journal articles? Eastern Economic Journal, 6 (3/4), 189-193.

Hamilton, D. P. (1990). Publishing by-And for?-The numbers. Science (New York, N.Y.), 250 (4986), 1331-1332. https://doi.org/10.1126/science.2255902

Huamaní, C.; Mayta-Tristán, P. (2010). Producción científica peruana en medicina y redes de colaboración, análisis del Science Citation Index 2000-2009. Revista Peruana de Medicina Experimental y Salud Pública, 27 (3), 315-325. https://doi.org/10.1590/S172646342010000300003

INEI (2017a). Censos Nacionales 2017. http://censo2017.inei.gob.pe/

INEI (2017b). Encuesta demográfica y de salud familiar -ENDES. https://www.inei.gob.pe/media/MenuRecursivo/publicaciones_digitales/Est/Lib1525/index.html

Kumar, S. (2015). Co-authorship networks: A review of the literature. Aslib Journal of Information Management, 67 (1), 55-73. https://doi.org/10.1108/AJIM09-2014-0116

Lorente Acosta, M. (2017). Tú haz la comida, que yo cuelgo los cuadros: Trampas y tramposos en la cultura de la desigualdad. Barcelona: Crítica.

Maliniak, D.; Powers, R.; Walter, B. F. (2013). The gender citation gap in international relations. International Organization, 67 (4), 889-922. https://doi.org/10.1017/ S0020818313000209

Maske, K. L.; Durden, G. C.; Gaynor, P. E. (2003). Determinants of scholarly productivity among male and female economists. Economic Inquiry, 41 (4), 555-564. https://doi.org/10.1093/ei/cbg027

Mcdowell, J. M.; Singell, L. D.; Stater, M. (2006). Two to Tango? gender differences in the decisions to publish and coauthor. Economic Inquiry, 44 (1), 153-168. https://doi.org/10.1093/ei/cbi065

Melin, G. (2000). Pragmatism and self-organization: Research collaboration on the individual level. Research Policy, 29 (1), 31-40. https://doi.org/10.1016/S00487333(99)00031-1
Mirick, R. G.; Wladkowski, S. P. (2018). Pregnancy, motherhood, and academic career goals: doctoral students' perspectives. Affilia, 33 (2), 253-269. https://doi.org/10.1177/0886109917753835

Mitchell, Sara McLaughlin, y Vicki L. Hesli. 2013. "Women Don't Ask? Women Don't Say No? Bargaining and Service in the Political Science Profession". PS: Political Science \& Politics 46 (02): 355-69. https:// doi.org/10.1017/S1049096513000073

Newman, M. E. (2010). Networks: An introduction. Oxford ; New York: Oxford University Press. https://doi. org/10.1093/acprof:oso/9780199206650.001.0001

Noorden, R. V. (2017). The science that's never been cited. Nature, 552, 162. https://doi.org/10.1038/ d41586-017-08404-0

(OCTS-OEI) Observatorio Iberoamericano de la Ciencia, la Tecnología y la Sociedad de la Organización de Estados Iberoamericanos. (2018). Las brechas de género en la producción científica iberoamericana. Disponible en https://observatoriocts.oei.org. ar/2018/10/12/n-09-las-brechas-de-genero-en-laproduccion-cientifica-iberoamericana/

Ozel, B.; Kretschmer, H.; Kretschmer, T. (2014). Co-authorship pair distribution patterns by gender. Scientometrics, 98 (1), 703-723. https://doi. org/10.1007/s11192-013-1145-y

Stvilia, B.; Hinnant, C. C.; Wu, S.; Worrall, A.; Lee, D. J. ; Burnett, K. ; ... Marty, P. F. (2017). Toward collaborator selection and determination of data ownership and publication authorship in research collaborations. Library \& Information Science Research, 39 (2), 8597. https://doi.org/10.1016/j.lisr.2017.03.004

Teele, D. L.; Thelen, K. (2017). Gender in the Journals: Publication Patterns in Political Science. PS: Political Science \&amp; Politics, 50 (2), 433-447. https:// doi.org/10.1017/S1049096516002985

Thege, B.; Popescu-Willigmann, S.; Pioch, R.; Badri-Höher, S. (Eds.). (2014). Paths to Career and Success for Women in Science. Wiesbaden: Springer. https://doi.org/10.1007/978-3-658-04061-1

Torres-Salinas, D. ; Muñoz-Muñoz, A. M. ; Jiménez-Contreras, E. (2011). Análisis bibliométrico de la situación de las mujeres investigadoras de Ciencias Sociales y Jurídicas en España. Revista Española de Documentación Científica, 34 (1), 11-28. https:// doi.org/10.3989/redc.2011.1.794

West, J. D.; Jacquet, J.; King, M. M.; Correll, S. J.; Bergstrom, C. T. (2013). The role of gender in scholarly authorship. PLOS ONE, 8 (7), e66212. https:// doi.org/10.1371/journal.pone.0066212

Zettler, H. R.; Cardwell, S. M.; Craig, J. M. (2017). The gendering effects of co-authorship in criminology \& criminal justice research. Criminal Justice Studies, 30 (1), 30-44. https://doi.or$\mathrm{g} / 10.1080 / 1478601 X .2016 .1265958$

Zuckerman, H.; Cole, J. R.; Bruer, J. T. (1991). The Outer Circle: Women in the Scientific Community. New Haven and London: Norton. 


\section{APÉNDICE 1}

Tabla A-I. Coautoría e impacto

\begin{tabular}{|c|c|c|c|c|c|c|}
\hline \multirow{3}{*}{ Indicadores } & \multirow{3}{*}{$\begin{array}{l}\text { Sin } \\
\text { coautoría }\end{array}$} & \multicolumn{5}{|c|}{ Coautoría } \\
\hline & & \multicolumn{2}{|c|}{ Por origen } & \multicolumn{3}{|c|}{ Por género } \\
\hline & & Nacional & Internacional & $\begin{array}{l}\text { Sólo } \\
\text { hombres }\end{array}$ & $\begin{array}{l}\text { Equipos } \\
\text { mixtos }\end{array}$ & $\begin{array}{l}\text { Sólo } \\
\text { mujeres }\end{array}$ \\
\hline Número de artículos & 132 & 529 & 233 & 223 & 502 & 32 \\
\hline \% total de artículos & 14.77 & 59.17 & 26.06 & 24.94 & 56.15 & 3.58 \\
\hline Promedio de citas & 0.6 & 0.97 & 1.88 & 1.26 & 1.27 & 0.56 \\
\hline $\begin{array}{l}\text { Número de citas del } \\
\text { artículo más citado }\end{array}$ & 14 & 41 & 50 & 28 & 50 & 5 \\
\hline $\begin{array}{l}\text { Número de artículos en } \\
\text { el top } 10 \%\end{array}$ & 0 & 56 & 36 & 31 & 59 & 1 \\
\hline $\begin{array}{l}\text { Proporción de artículos } \\
\text { en el top } 10 \%\end{array}$ & 0 & 10.59 & 15.45 & 13.90 & 11.75 & 3.12 \\
\hline
\end{tabular}

\section{APÉNDICE 2}

Tabla A-II. Características de los autores con mayor productividad en la componente gigante.

\begin{tabular}{|c|c|c|c|c|c|c|}
\hline Investigador/a & Sexo & $\begin{array}{l}\text { Instituciones a las } \\
\text { que estuvo afiliado }\end{array}$ & Periodo & $\begin{array}{l}\text { Tipos de } \\
\text { coautoría }\end{array}$ & $\begin{array}{l}\text { Número } \\
\text { de } \\
\text { artículos }\end{array}$ & $\underset{H}{\text { Índice }}$ \\
\hline Pacheco-Torres, Víctor & masculino & UNMSM (Perú)* & 2007-2017 & $\mathrm{N}: 20 ; \mathrm{I}: 4$ & 24 & 4 \\
\hline León, Blanca & femenino & $\begin{array}{l}\text { University of Texas } \\
\text { at Austin (USA); } \\
\text { UNMSM (Perú)* }\end{array}$ & 2004-2017 & $\begin{array}{l}\text { N: 3; I: } 13 ; \\
\text { S: } 2\end{array}$ & 18 & 2 \\
\hline Cano, Asunción & masculino & UNMSM (Perú)* & 2003-2016 & $\mathrm{N}: 15 ; \mathrm{I}: 3$ & 18 & 4 \\
\hline Tantaleán-V., Manuel & masculino & $\begin{array}{l}\text { UPCH (Perú)**; } \\
\text { UNALM (Perú)***; } \\
\text { UNMSM (Perú)* }\end{array}$ & 2004-2011 & $\mathrm{N}: 15 ; \mathrm{I}: 3$ & 18 & 2 \\
\hline Millán, Betty & femenino & UNMSM (Perú)* & $2008-2015$ & N: $10 ;$ I: 6 & 16 & 2 \\
\hline Kahn, Francis & masculino & $\begin{array}{l}\text { UNMSM (Perú)*; } \\
\text { Institut de } \\
\text { Recherche pour le } \\
\text { Developpement, } \\
\text { (Perú, Francia, Brasil) }\end{array}$ & $2008-2015$ & $\begin{array}{c}\mathrm{N}: 10 ; \mathrm{I}: 4 \\
\mathrm{~S}: 1\end{array}$ & 15 & 2 \\
\hline Aquino, Rolando & masculino & UNMSM (Perú)* & 2005-2017 & $\begin{array}{c}\mathrm{N}: 12 ; \mathrm{I}: 1 ; \\
\mathrm{S}: 1\end{array}$ & 14 & 3 \\
\hline Gómez-Puerta, Luis & masculino & UNMSM (Perú)* & $2005-2017$ & N: $6 ;$ I: $3 ;$ S: 4 & 13 & 2 \\
\hline $\begin{array}{l}\text { Rodríguez Rodríguez, } \\
\text { Eric }\end{array}$ & masculino & $\begin{array}{l}\text { Universidad Nacional } \\
\text { de Trujillo (Perú); } \\
\text { Museo de Historia } \\
\text { Natural (Chile) }\end{array}$ & $2005-2013$ & $N: 5 ; \mathrm{I}: 8$ & 13 & 3 \\
\hline Ceroni-Stuva, Aldo & masculino & UNALM (Perú)*** & 2004-2015 & $\mathrm{N}: 10 ; \mathrm{I}: 1$ & 11 & 1 \\
\hline Pino Gaviño, José & masculino & UNMSM (Perú)* & 2004-2013 & $\mathrm{N}: 11$ & 11 & 1 \\
\hline Ramirez, Rina & femenino & UNMSM (Perú)* & 2009-2017 & $\mathrm{N}: 7 ; \mathrm{I}: 3$ & 10 & 2 \\
\hline Sánchez Vega, Isidoro & masculino & $\begin{array}{l}\text { Universidad Nacional } \\
\text { Cajamarca (Perú) }\end{array}$ & $2006-2006$ & $\mathrm{~N}: 2 ; \mathrm{I}: 8$ & 10 & 1 \\
\hline
\end{tabular}

Leyenda: N: Nacional, I: Internacional, S: Sin Coautoría, *Universidad Nacional Mayor de San Marcos, ** Universidad Peruana Cayetano Heredia, *** Universidad Nacional Agraria La Molina. 